\title{
RegEx Übersicht
}

Tab. 34.1 RegEx Übersicht

\begin{tabular}{|c|c|c|c|}
\hline RegEx String & Erklärung & RegEx String & Erklärung \\
\hline . & $\begin{array}{l}\text { Alle Zeichen außer Codes für } \\
\text { „nächste Zeile“ }\end{array}$ & $(?: a b c)$ & $\begin{array}{l}\text { Gruppe, die nicht vorhanden } \\
\text { sein darf }\end{array}$ \\
\hline a & Zeichen a & $\backslash 1$ & Referenz zur Gruppe 1 \\
\hline xy & String xy & $a^{*} b+c ?$ & $\begin{array}{l}0 \text { oder mehr Mal a, } 1 \text { oder } \\
\text { mehr Mal b, } 0 \text { - oder } 1 \text {-mal c }\end{array}$ \\
\hline$\backslash \mathrm{w} \backslash \mathrm{d} \backslash \mathrm{s}$ & Ein Wort, eine Zahl, ein Space & $\mathrm{a}\{5\} \mathrm{b}\{3 ;\}$ & $\begin{array}{l}\text { Genau fünf Mal a, drei oder } \\
\text { mehr Mal b }\end{array}$ \\
\hline$\backslash \mathrm{W} \backslash \mathrm{D} \backslash \mathrm{S}$ & $\begin{array}{l}\text { Kein Wort, keine Zahl, kein } \\
\text { Space }\end{array}$ & $\mathrm{a}\{1 ; 3\}$ & Zwischen ein und drei Mal a \\
\hline$[a b c]$ & Eines der Zeichen a, b, oder c & alb & a oder b \\
\hline$[\wedge \mathrm{abc}]$ & Keines der Zeichen $\mathrm{a}, \mathrm{b}$, oder $\mathrm{c}$ & ablyz & ab oder yz \\
\hline$[\wedge \mathrm{ax}-\mathrm{z}]$ & Ein Zeichen ohne a, $\mathrm{x}, \mathrm{y}, \mathrm{z}$ & $\mathrm{i}$ & $\begin{array}{l}\text { Groß-/Kleinschreibung } \\
\text { ignorieren }\end{array}$ \\
\hline$[\mathrm{a}-\mathrm{k}]$ & Ein Zeichen zwischen a und $\mathrm{k}$ & $\wedge \$$ & Start und Ende einer Zeile \\
\hline$[\mathrm{ab}-\mathrm{e}]$ & Entweder a, b, c, d oder e & a.b & $\begin{array}{l}\text { a gefolgt von einem anderen } \\
\text { Buchstaben gefolgt von b }\end{array}$ \\
\hline$\wedge a b c \$$ & Start und Ende des Strings abc & a. $* b$ & $\begin{array}{l}\text { a gefolgt von mehreren } \\
\text { Buchstaben gefolgt von b }\end{array}$ \\
\hline$\backslash . \backslash * \backslash \backslash$ & Eingabe von Spezial-Zeichen & {$[a b]+$} & a, b, aa, ab, aaaba, abab, etc. \\
\hline$\backslash \mathrm{b} \backslash \mathrm{t} \backslash \mathrm{n} \backslash \mathrm{r}$ & $\begin{array}{l}\text { Backspace, Tabulator, nächste } \\
\text { Zeile, Zeilenvorschub }\end{array}$ & $(a b c)$ & Gruppieren \\
\hline
\end{tabular}

